\title{
具有非线性阻尼与软弹簧系统的极限环 及其对机珹振动的应用
}

\author{
李继棌 \\ (昆明工学院数学教研室)
}

51. 本文研究控制方程

$$
\ddot{x}+\left(a|\dot{x}|^{\lambda}+b|\dot{x}|^{\mu}+c\right) \dot{x}+\left(x-\beta^{2} x^{3}\right)=0,
$$

其中, $0<\lambda<1, \mu>1+\lambda, \beta>0$.（1.1）式是工程实践中提出的非线性振动模型. 当 $\beta=0,1+\lambda+\mu<2$ 时, 已在文献 [1] 中讨论过. 文献 [2] 用图解法讨论过 (1.1) 式的一 个具体数值例子. 文献 [3] 曾研究 (1.1) 式中 $a$ 或 $b$ 等于零时, 位移项有参数謜的极限环 响应. 为研究 (1.1) 式我们首先一般地讨论方程组

$$
\frac{d x}{d t}=\varphi(y)-F(x), \frac{d y}{d t}=-x,
$$

其中, $\varphi(y)-y-\beta^{2} y^{3}$ 对应于 (1.1) 式中的软弹簧力, 而 $F(x)$ 为较广泛的函数类. 特别当 (1.2) 式中 $F(x)-(|x|-q) x$ 时, 就是 $\mathrm{Krall}^{[4]}$ 所研究过的方程. 当 $y \varphi(y)>0$ 时, 比 (1.2) 式更广泛的方程组

$$
\frac{d x}{d t}=\varphi(y)-F(x), \frac{d y}{d t}=-g(x),
$$

的研究已有文献 [5,6] 等工作. 本文推广了张芷芬 ${ }^{[6]}$ 的某些结果到软弹簧系统, 得到 $\$ 2$ 中 方程组 (2.1) 存在多个极限环的一组充分条件, 将 52 的结果用于方程 (1.1) 所对应的方程组 (1.2), 我们讨论了方程 (1.1) 存在或不存在极限环的判别准则, 52 的结果包含了文献 [4] 的 方程.

52. 给定微分方程组

$$
\left\{\begin{array}{l}
\frac{d x}{d t}=\varphi(y)-F(x)=y-\beta^{2} y^{3}-F(x), \\
\frac{d y}{d t}=-x,
\end{array}\right.
$$

文献 [6] 引人的“两条曲线 $n$ 重互相包含”概念可推广为

定义 2.1 两条曲线 $y=F_{1}(x), y=F_{2}(x)$ 叫做在区间 $[a, b]$ 上 “ $n$ 重互相包含后分 离” $(n=0,1,2, \cdots)$, 如果满足条件

1. $y=F_{1}(x)$ 与 $y-F_{2}(x)$ 在 $[a, b]$ 上 “ $n$ 重互相包含”, 即满足下述条件

(i) $y=F_{1}(x)$ 与 $y=F_{2}(x)$ 相交于 $n+2$ 个点 $\left(a_{i}, b_{i}\right), i=1,2, \cdots, n+2 ; a=$ $a_{1}<a_{2}<\cdots<a_{n+1}<a_{n+2}=b$.

本文1981年9月14 日收到。 


$$
(-1)^{i+1}\left(F_{2}(x)-F_{1}(x)\right) \geqslant 0, \quad a_{i}<x<a_{i+1},
$$

$i=1,2, \cdots, n+1$.

(ii) 存在 $\tau_{i+1}^{i}, \xi_{i+1}^{i} \in\left[a_{i+1}, a_{i+2}\right], \xi_{i+1}^{i} \geqslant \tau_{i+1}^{i}$, 令 $\Delta_{i+1}^{i}-\tau_{i+1}^{i}-a_{i}, \Delta_{i+1}^{i}-\xi_{i+1}^{j}-a_{i}$, $\boldsymbol{\gamma}_{i+1}-\max _{i=1,2}\left(\xi_{i+1}^{j}+\Delta_{i+1}^{i}\right)$, 则有

a) $(-1)^{i+i} F_{i}(x) \geqslant 0$, 当 $x \in\left[\tau^{i},+1, r_{i+1}\right] \subset\left[a_{i+1}, a_{i+2}\right]$,

b) $(-1)^{l}\left[(-1)^{s} F_{s}(x)+(-1)^{\imath} F_{l}\left(x+\Delta_{i+1}^{l}\right] \geqslant 0 \neq 0\right.$.

当 $x \in\left[a_{i}, \tau_{i+1}^{s}\right]$, 其中 $s \neq l, s, i, l=1,2, i=1,2, \cdots, n$.

2. 在 $[b,+\infty]$ 上, $y=F_{1}(x)$ 与 $y=F_{2}(x)$ 单调, $F_{1}(+\infty)=(-1)^{n} \infty, F_{2}(+\infty)=$ $(-1)^{n+1} \infty$.

对方程组 (2.1) 假设满足条件

(I) $F(x) \in C^{1}(-\infty,+\infty)$, 保证初值问题解存在唯一.

(II) 令 $F_{1}(x)=F(x), F_{2}(x)=F(-x)$, 曲线 $y=F_{1}(x)$ 与 $y-F_{2}(x)$ 在 $[0, b]$ 上 “ $n$ 重互相包含后分离”, 记 $f(x)-F^{\prime}(x)$ 不妨设 $f(0)<0$.

容易证明，(2.1) 式在相平面上有三个有限远奇点 $O(0,0), A(0,1 / \beta), B(0,-1 / \beta)$. 若 $f(0)<0$, 点 $O$ 是不稳定的初等奇点, 点 $A$ 与 $B$ 为鞍点.

$$
\text { 令 } \begin{aligned}
m_{i+1}^{i} & =\max _{x \in\left[a_{i+1} a_{i+2}\right]}\left|F_{i}(x)\right|, j=1,2, \cdots, n, \\
m_{i+1} & =\max _{i=1,2} m_{i+1}^{i},
\end{aligned}
$$

考察方程组 (2.1) 的无穷等倾线

$$
y-\beta^{2} y^{3}-F(x)-\Psi(x, y)=0 .
$$

引理 2.1 (i) 若 $m_{n+1}<\frac{2}{3 \sqrt{ } 3 \beta} \approx 0.385 \beta^{-1}$ 则曲线方程 (2.2) 没有重点.

(ii) 若 $m_{n+1}^{i}<\frac{2}{3 \sqrt{3} \beta}-m_{n+1}^{i}, i \neq i, i, j-1,2$, 又成立着

$$
(-1)^{n}\left[(-1)^{s} F_{s}(x)+(-1)^{l} F_{l}\left(x+\bar{\Delta}_{n+1}^{l}\right)\right]>0 \text {, 当 } x \in\left[a_{n}, \tau_{n+1}^{s}\right] \text {, }
$$

又 $F_{j}^{\prime}(x)=f_{j}(x)$. 当 $x \in\left[a_{n+1}, a_{n+2}\right]$ 时, 零点唯一, 其中 $s, j, l=1,2, s \neq l$, 且在该零点 $f^{\prime}(x) \neq 0$, 则曲线方程 (2.2) 恰有一个二重点.

(iii) 若 $m_{n+1}^{1}=m_{n+1}^{2}=\frac{2}{3 \sqrt{3} \beta}$, 而且 (2.3) 式与 $f_{j}(x)$ 的上述条件成立, 则曲线方程 (2.2) 恰有两个二重点.

通过研究鞍点分界线的走向与无穷等倾线方程 (2.2) 的关系,并利用比较定理与文献 [6] 得到下面的三个定理. 首先引人记号

$$
\begin{aligned}
& \alpha=\max \left(\left|\alpha_{1}\right|,\left|\alpha_{2}\right|\right), \quad \alpha_{1}=F^{-1}\left(\frac{2}{3 \sqrt{3} \beta^{\prime}}\right), \quad \alpha_{2}=F^{-1}\left(-\frac{2}{3 \sqrt{3} \beta}\right), \\
& \gamma=\min \left(\left|\gamma_{1}\right|,\left|\gamma_{2}\right|\right), \quad \gamma_{1}=\frac{1}{\sqrt{2} \beta}\left(1-4 m_{n+1}^{1} \beta\right)^{\frac{1}{2}}, \\
& \gamma_{2}=-\frac{1}{\sqrt{2} \beta}\left(1-4 m_{n+1}^{2} \beta\right)^{\frac{1}{2}} .
\end{aligned}
$$

定理 2.1 若方程组 (2.1) 中函数 $F(x)$ 满足条件 (I)、(II), 并对适当小的 $\beta$, 成立着 
(III) $a<r$, 则方程组 (2.1) 在带域 $|x|<\alpha$ 中至少存在 $n+1$ 个极限环.

再引入记号

$l_{n+1}-\max _{j=1.2} l_{n+1}^{\prime}$, 其中 $-l_{n+1}^{2}$ 与 $l_{n+1}^{1}$ 分别为 $f(x)$ 在 $\left[-a_{n+2},-a_{n+1}\right],\left[a_{+1}, a_{n+2}\right]$ 内的 点.

$$
\begin{aligned}
& \gamma_{1}^{*}=\frac{1}{\sqrt{2} \beta}\left(1-4 m_{m}^{1} \beta\right)^{\frac{1}{2}}, \gamma_{2}^{*}=-\frac{1}{\sqrt{2 \beta}}\left(1-4 m_{n}^{2} \beta\right)^{\frac{1}{2}}, \\
& \gamma^{*}=\min _{j=1.2}\left|\gamma_{i}^{*}\right| .
\end{aligned}
$$

它理 2.2 若微分方程组 (2.1) 中函数 $F(x)$ 满足条件 (I), (II) 及引理 2.1 (iii) 的条 件, 并成立着 (III) $r^{*}>l_{n+1}$, 则在带域 $|x|<l_{n+1}$ 中至少存在方程 (2.1) 的 $n$ 个极限环.

定珽 2.3 若微分方程组 (2.1) 的右端函数 $F(x)$ 满足 (I)、(II) 及引理 2.1 (ii) 的条件, 且成立着 (IV) $\gamma_{2}<\alpha_{2}$, 则在带域 $\alpha_{2}<x<l_{n+1}^{\prime}$ 中至少存在方程 (2.1) 的 $n$ 个极限环.

53. 记方程组 (2.1) 中 $F(x)$ 为

$$
F(x)=\frac{F(x)+F(-x)}{2}+\frac{F(x)-F(-x)}{2}-E(x)+\theta(x),
$$

显然 $E(x)$ 为偶函数, $\theta(x)$ 为奇函数.

引理 3.1 设 $E(x)$ 常号.

(i) 方程组

$$
\frac{d x}{d t}=y-\beta^{2} y^{3}-E(x), \frac{d x}{d t}=-x,
$$

存在转点分界线环, 环内充满方程 (3.1) 的包围原点的闭轨线.

(ii) 若 $x>0, \theta(x)$ 常号,则方程 (2.1) 无周期解.

(iii) 若 $x>0, \theta(x)$ 变号, $x=a_{2}$ 为 $\theta(x)$ 的第一个正点, 且过该零点 $\theta(x)$ 变号, 则方 程 (2.1) 如存在周期解, 该解必同时与 $x=a_{2}, x=-a_{2}$ 相交.

以下讨论机械振动系统

$$
\left\{\begin{array}{l}
\frac{d x}{d t}=y-\beta^{2} y^{3}-\left(a|x|^{2}+b|x|^{\mu}+c\right) x, \\
\frac{d y}{d t}=-x,
\end{array}\right.
$$

记 $F(x)-\left(a|x|^{\alpha}+b|x|^{\mu}+c\right) x, f_{1}(x)=a|x|^{\alpha}+b|x|^{\mu}+c, F^{\prime}(x)=f(x)$. 显然 $F(x)$ 为奇函数,引理 3.1 中 $E(x)=0, F(x)=\theta(x)=0$.

定理 3.1 方程组 (3.2) 不存在周期解,若以下条件之一成立

(i) $a b c \neq 0, a, b, c$ 同号;

(ii) $a, b, c$ 之一为零,其余两个同号;

(iii) $a b<0, b c<0, f_{1}\left(l^{*}\right)\left\{\begin{array}{l}\leqslant 0 \text { 当 } a>0 \text { 时, } \\ \geqslant 0 \text { 当 } a<0 \text { 时; }\end{array}\right.$

其中 $l^{*}-\left[-\frac{a \lambda}{b \mu}\right]^{\frac{1}{\mu-\lambda}}$ 为 $y=f_{1}(x)$ 在 $(0,+\infty)$ 上的极值点.

(iv) $F(x)$ 的第一个正点 $a_{2} \geqslant 1 / \sqrt{2} \beta$.

定理 3.2 设方程组 (3.2) 中 $F(x)$ 满足条件 


$$
\frac{1}{\sqrt{2} \beta}\left(1-4\left|F\left(l_{1}\right)\right| \beta\right)^{\frac{1}{2}}>F^{-1}\left(\frac{2}{3 \sqrt{3} \beta}\right),
$$

其中 $l_{1}$ 为 $f(x)$ 的第一个正䗙点, 又设成立着

(i) $b=0, a>0, c<0$ 或 (ii) $b>0, c<0$;

则方程组 (3.2) 至少存在一个极限环.

定理 3.3 设方程组 (3.2) 中 $a>0, b<0, c<0$ 又

(i) 在区间 $\left[a_{2}, a_{3}\right]$ 上存在点 $\tau_{2}$, 使得

$$
F(x)+F\left(x+\tau_{2}\right)>0 \text {, 当 } x \in\left[0, \tau_{2}\right] \text { 时, }
$$

(ii) $\frac{1}{\sqrt{2} \beta}\left(1-4 F\left(l_{2}\right) \beta\right)^{\frac{1}{2}}>\left|F^{-1}\left(\frac{2}{3 \sqrt{3} \beta}\right)\right|$,

则方程 (3.2) 至少存在两个极限环.

定理 3.4 若方程组 (3.3) 中 $a>0, b<0, c<0$, 又定理 3.3 的条件 (i) 成立, 且满足

(ii) $\frac{1}{\sqrt{2} \beta}\left(1-4\left|F\left(l_{1}\right)\right| \beta\right)>l_{2}$, (iii) $F\left(l_{2}\right)-\frac{2}{3 \sqrt{3} \beta}$,

则方程 (3.2) 至少存在一个极限环.

例如文献 [2] 的数值例子

$$
\frac{d x}{d t}=y-0.1 y^{3}-\left(1.2|x|^{0.04}-|x|^{1.9}-0.2\right) x, \frac{d y}{d t}=-x,
$$

满足定理 3.3 的条件, 至少存在两个极限环.

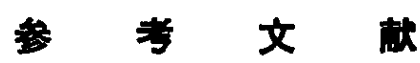

[1] 本继林,东北师大学报(自然科学版), 1981, 2: 15-17.

[2]尿维德, 昆明工学院学报, 1981, 1: 1-7.

[3] Hsu, C. S.,J. Appl. Mech., 42 (1975), 176-182.

[4] Krall, G, Atti Acc. Naz. Lincei. Rend. Sci. Fis. Mat., 5 (1948), 8:197-203.

[5] Рычков Г. С., Сибир. Матем. Журнал, 7 (1966), 6:1425-1431.

[6] 张芷芬,关于一类非线性方程存在多个极限环的条件，大连全国常微分方程会议交流资料，1981，8. 